\title{
La cultura organizacional como base del cumplimiento de objetivos estratégicos organizacionales
}

\author{
Alfredo Augusto Paz Subillaga ${ }^{1}$ \\ Rafael Núñez ${ }^{2}$
}

\section{RESUMEN}

Para los gestores de capital humano en las organizaciones, es vital poder contar con herramientas, factores y aspectos que les permitan alcanzar los objetivos propuestos, tanto de su área como de la organización en general. Esto conlleva a que los gestores deban y sepan acoplar y comprender variables de y en su propia gestión que permitan poder llegar a buen puerto con lo que una organización desea avanzar en su desarrollo.

Tal es el caso de la cultura organizacional, que a su vez es una herramienta intangible en muchos aspectos, pero es tan poderosa que se puede sentir su peso dentro del ambiente organizacional. Igualmente, está el establecimiento, desarrollo y consecución de objetivos organizacionales estratégicos que son apoyados en gran parte por esta cultura organizacional. El enlace de estas dos variables da como resultado una cultura de cumplimiento por parte de los colaboradores en una organización.

El objetivo de esta investigación es demostrar la importancia que existe entre el enlace de estas dos variables, que a su vez pueda permitir la sensibilización entre los gestores de recursos humanos y la cúpula organizacional. Es una investigación de carácter descriptivo y muestra en sus principales resultados que la atención y seguimiento de las variables tratadas por los gestores de recursos humanos en las empresas metalmecánicas son aspectos que pueden ayudar a alcanzar objetivos estratégicos de una manera más concreta y adecuada.

Esta investigación es de tipo cuantitativa con propósito descriptivo, en la cual se trabajó una muestra total de 16 gerentes de recursos humanos de empresas metal-

\footnotetext{
${ }^{1}$ Estudiante de Doctorado en Ciencias de la Investigación, Universidad Mariano Gálvez de Guatemala: pazsubillaga@gmail.com

${ }^{2}$ Asesor, Facultad de Ciencias Económicas, UNAH: rafael.nunez@unah.edu.hn
} 
mecánicas, consideradas grandes, que cuentan con más de mil empleados de la Ciudad de Guatemala.

Entre las conclusiones más importantes de esta investigación están: la atención y manejo de la cultura organizacional por parte de los gestores de capital humano y de la cúpula de las organizaciones, lo que permite cumplir muchos objetivos como ahorrar costos laborales y mantener una identificación del personal hacia su organización.

Se necesita que la misión de los gestores del capital humano esté mucho más enfocada en la parte estratégica, pues esto permite que su gestión tenga mucha más atención por parte de la cúpula organizacional, a tener solamente una gestión operativa sin relevancia.

Al momento de enlazar estas dos variables se requiere compromiso por parte no solo del área de capital humano, sino todo un compromiso a nivel general para que esto les permita ser una organización proactiva y no reactiva.

Palabras clave: investigación, cultura organizacional, objetivos estratégicos, organizaciones proactivas y reactivas, presupuesto, gestión de capital humano.

\section{ABSTRACT}

For managers of human capital in organizations, it is vital to have tools that allow factors and aspects to achieve the objectives of both the area and the organization in general. This entails that these managers should engage and understand and know variables and their own management that allow to come to fruition with what an organization wants to advance its development.

Such is the case of organizational culture, which in turn is a tool in many intangible aspects but is so powerful that you can feel its weight within the organizational environment. And on the other hand, it is the establishment, development and achievement of strategic organizational objectives, which are supported in large part by the organizational culture.

The link of these two variables results in a culture of compliance by employees in an organization. 
The objective of this research is to demonstrate the importance of the link between these two variables and which in turn can allow awareness among human resource managers and organizational leadership. Is a descriptive study and its main results shown in the care and monitoring of variables addressed in the human resource managers in the metalworking companies are aspects that can help achieve strategic objectives in a more concrete and properly.

Keywords: research, organizational culture, strategic objectives, proactive and reactive organizations, financial, human capital management. 


\section{INTRODUCCIÓN}

Toda empresa o institución tiene una cultura organizacional, aunque esta puede ser alta, baja o media; sin embargo, ¿cómo se puede saber realmente la dimensión de lo que significa alta, baja o media en una empresa? La respuesta es muy básica, ya que dependerá de cómo y con qué actitud se puedan afrontar situaciones determinadas por parte del personal de una empresa para poder saber esto.

Un parámetro fundamental para saber la respuesta es el cumplimiento de objetivos estratégicos organizacionales, puesto que si estos se cumplen de manera alta, media o baja, así será el reflejo en la cultura de la organización, pues aspectos actitudinales, de identificación hacia la organización, valores (entre ellos el respeto, uno de los más importantes para lograr buenos resultados en una empresa), comunicación en doble vía, libertad de expresión, competitividad, trabajo en equipo, compromiso y muchos otros factores, son vitales para poder lograr alcanzar objetivos.

Esto por sí solo no puede llegar a una empresa, pues el grupo de personas que la forman, también, a su vez, forman una cultura que determina un sello específico 0 único en una organización, ya que dependerá de cómo se vaya desarrollando esa cultura para que se puedan lograr metas establecidas por la cúpula de la empresa 0 institución y así esto pueda ser parte de ese "día a día" que se vive en el mundo laboral. Es necesario que todos los colaboradores de una empresa sepan hacia dónde va la organización y, sobre todo, qué papel desempeñan los empleados para lograr esos objetivos.

Existen organizaciones reactivas que cuando se enteran de que su competidor ha desarrollado cierta estrategia, ellos responden a la misma; en muchas ocasiones de manera desordenada e inconclusa. También existen otras organizaciones que son proactivas, son las que dan el primer paso en el mercado y van hacia adelante para tratar de lograr la meta en primer lugar. Pero, esto no es solo responsabilidad de los directivos, sino que detrás de ellos va un grupo de colaboradores que sienten y aportan para que la empresa sea proactiva, están preparados para las contingencias y cambios que los mercados exigen. Todo esto sucede porque internamente el capital humano y la organización en sí misma tienen una cultura fuerte, robusta y una agilidad que ayuda a que los objetivos se logren de mejor manera.

La agilidad consiste en generar esas condiciones organizacionales que permitan evitar la congestión y los cuellos de botella que inhiben la implementación o cumpli- 
miento de los objetivos estratégicos organizacionales (Kavacevic y Reynoso, 2010).

La presente investigación se desarrolló en el área de las empresas metalmecánicas de la Ciudad de Guatemala, tomando en cuenta que por aspectos demográficos y de comercio, estas empresas se encuentran en su mayoría en la capital, en donde se ha se desarrolla la mayor parte de la actividad económica y de trabajo para el país.

\section{METODOLOGÍA}

El tipo de estudio desarrollado para esta investigación fue cuantitativo con un enfoque descriptivo, pues el objetivo de la investigación versa sobre determinar la importancia que existe entre el enlace de la cultura organizacional y el cumplimiento de los objetivos estratégicos organizacionales; a su vez, que esto permita la sensibilización entre los gestores de recursos humanos y la cúpula organizacional.

El ámbito de estudio fue la ciudad capital de Guatemala y sus 16 empresas metalmecánicas más grandes (de mil empleados y más), las que, por supuesto, cuentan con procesos de provisión debidamente establecidos.

La muestra está conformada por las 16 empresas metalmecánicas y sus 16 gerentes de recursos humanos. Se diseñó un instrumento con preguntas dicotómicas enfocadas a obtener la información necesaria y, por último, una pregunta abierta para que se adhirieran los comentarios extras que quieran los entrevistados, las cuales no tendrían tabulación por ser comentarios muy generales o sugerencias al tema de investigación.

En las empresas metalmecánicas encuestadas por medio de sus gerentes de recursos humanos, se pudo constatar que al menos el $75 \%$ de estas empresas sí tiene mediciones de factores de la cultura organizacional dentro de su ambiente. Un $25 \%$ que no atiende estas mediciones, por lo cual es un porcentaje importante, tomando en cuenta que son empresas que cuentan con al menos mil empleados. 
Gráfico 1. Medición de la cultura organizacional

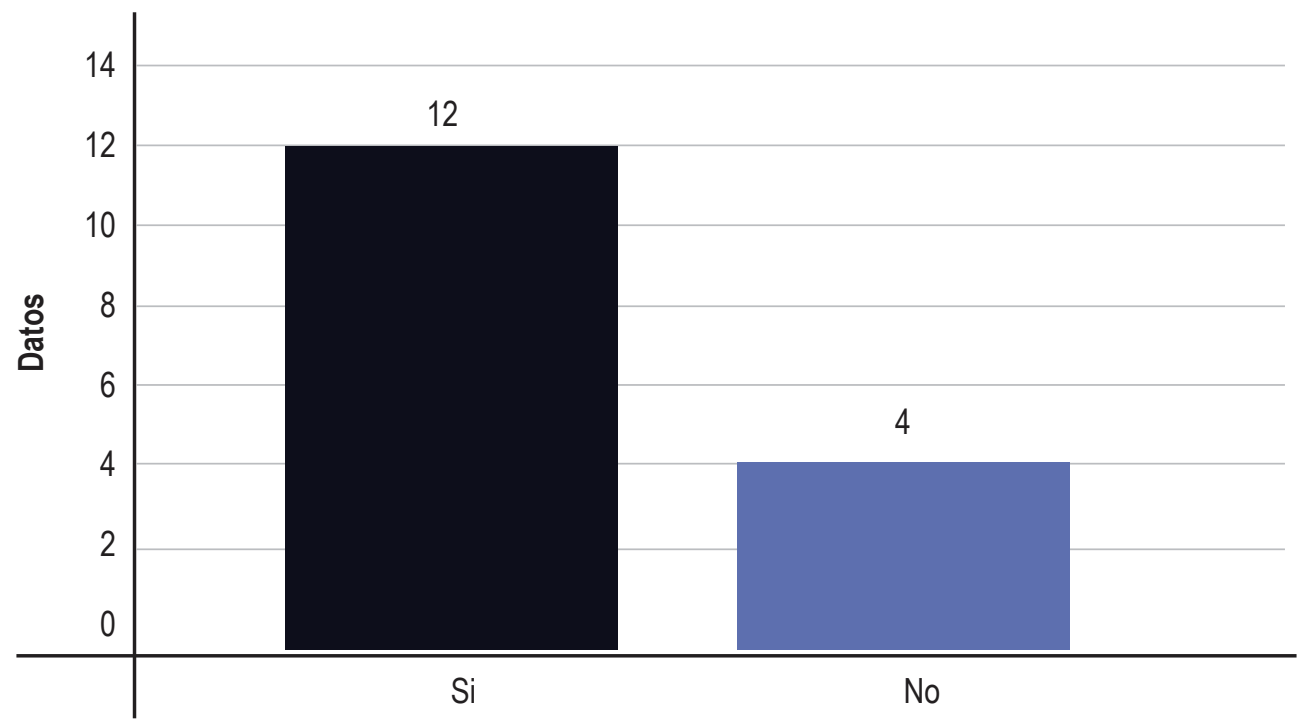

Fuente: elaboración propia.

\section{Gráfico 2. Objetivos estratégicos}

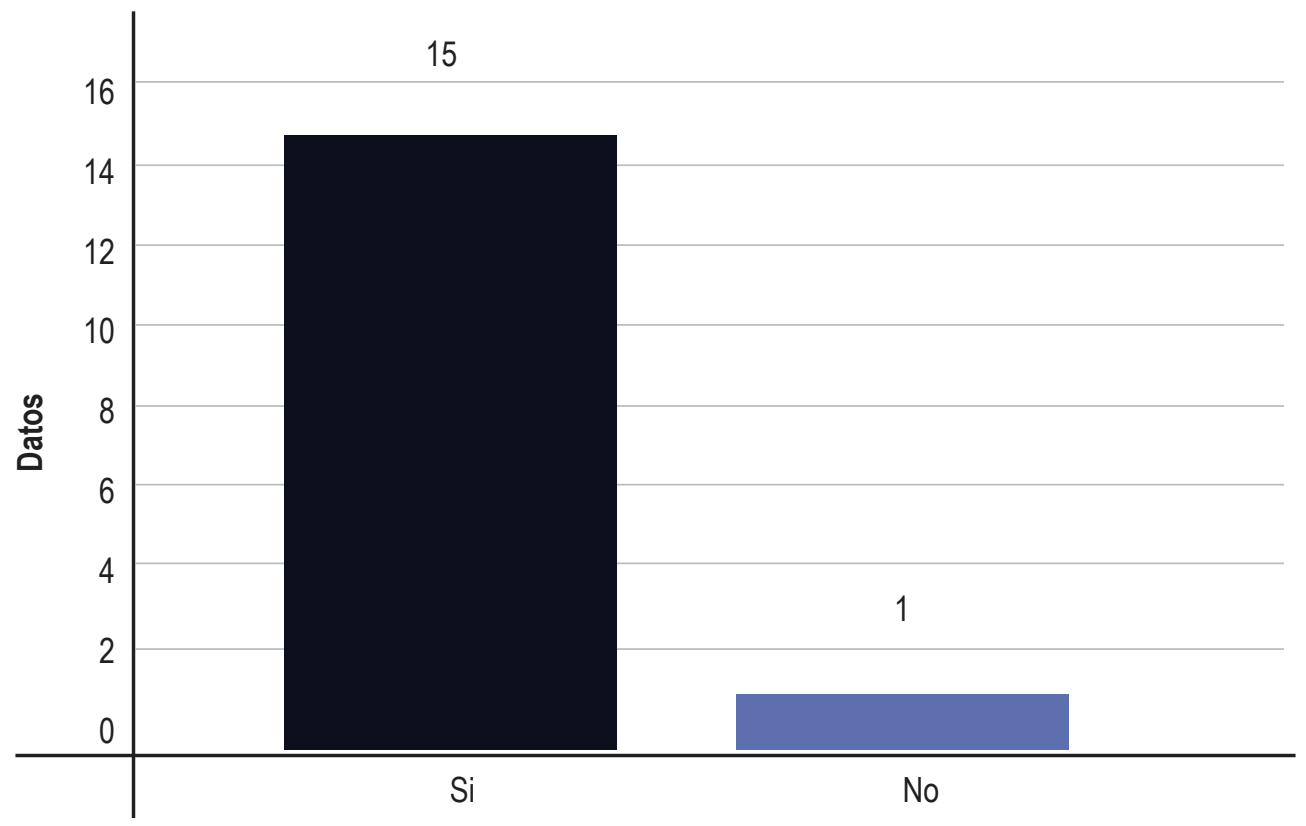

Fuente: elaboración propia. 
Definitivamente, el $94 \%$ de las empresas encuestadas tiene objetivos estratégicos establecidos dentro de su accionar empresarial. Es importante mencionar que dentro de ese establecimiento de objetivos se suman los generales y específicos por área, ya que representan una parte importante de cada objetivo general para poder tener una concatenación importante con respecto a lo que cada área debe realizar para su logro.

\section{RESULTADOS}

Dentro de los aspectos que se correlacionan en esta investigación está determinar cómo se relacionan las variables cultura organizacional y objetivos estratégicos, pero sobre todo, cómo los gestores de recursos humanos y la directiva de las empresas se alinean para poder hacer efectivo este amalgamiento y así llegar a un mismo cumplimiento.

La investigación arroja información importante según la cual los gerentes entrevistados muestran interés, en algunos casos sobre el amarre de las variables, pero existen aspectos como los financieros o presupuestarios, que afectan esas uniones, pues en ocasiones no existe algún tipo de presupuesto para poder aplicar un programa de motivación o lealtad hacia los colaboradores, lo cual afecta el desempeño laboral y, a su vez, llama a la no-consecución de objetivos o a su tardía consecución, lo que representa en su momento pérdidas monetarias para la empresa.

\section{Gráfico 3. Seguimiento a elementos de cultura organizacional por parte de recursos humanos}

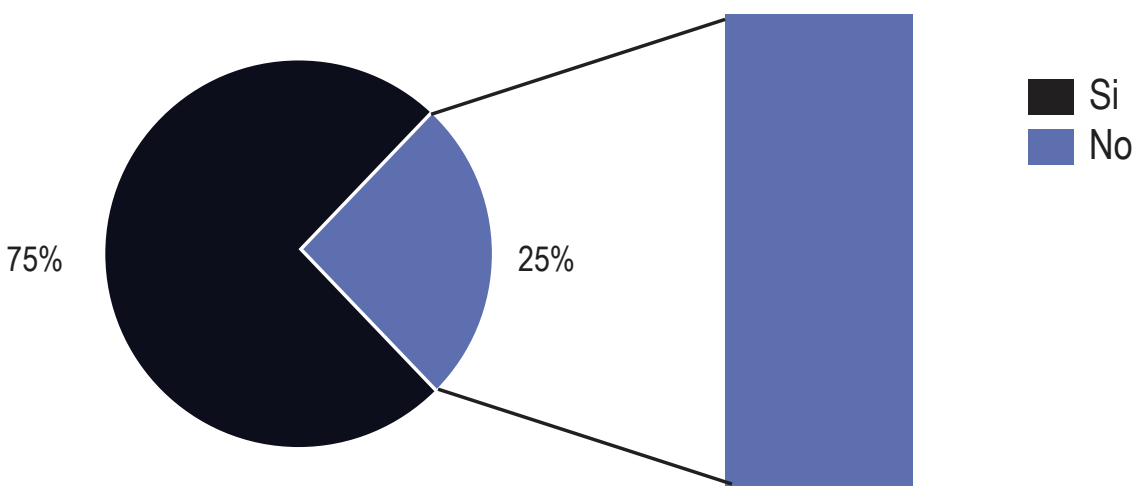

Fuente: elaboración propia. 
En las entrevistas sostenidas con los gestores de recursos humanos, se pudo constatar que al menos el $25 \%$ de los entrevistados no dan seguimiento a elementos de la cultura organizacional, es decir, no miden el clima organizacional; en algunos casos no dan seguimiento al tema de los valores institucionales, otros no verifican si la comunicación informal va disminuyendo en el grupo de colaboradores y otros. Esto demuestra que cuando los objetivos estratégicos se dan a conocer a los colaboradores, pueda que a algunos llegue el mensaje, pero no será recibido de la mejor manera y, por ende, su cuota de contribución para lograr los objetivos será mínima o nula.

\section{Gráfico 4. Cumplimiento de objetivos estratégicos}

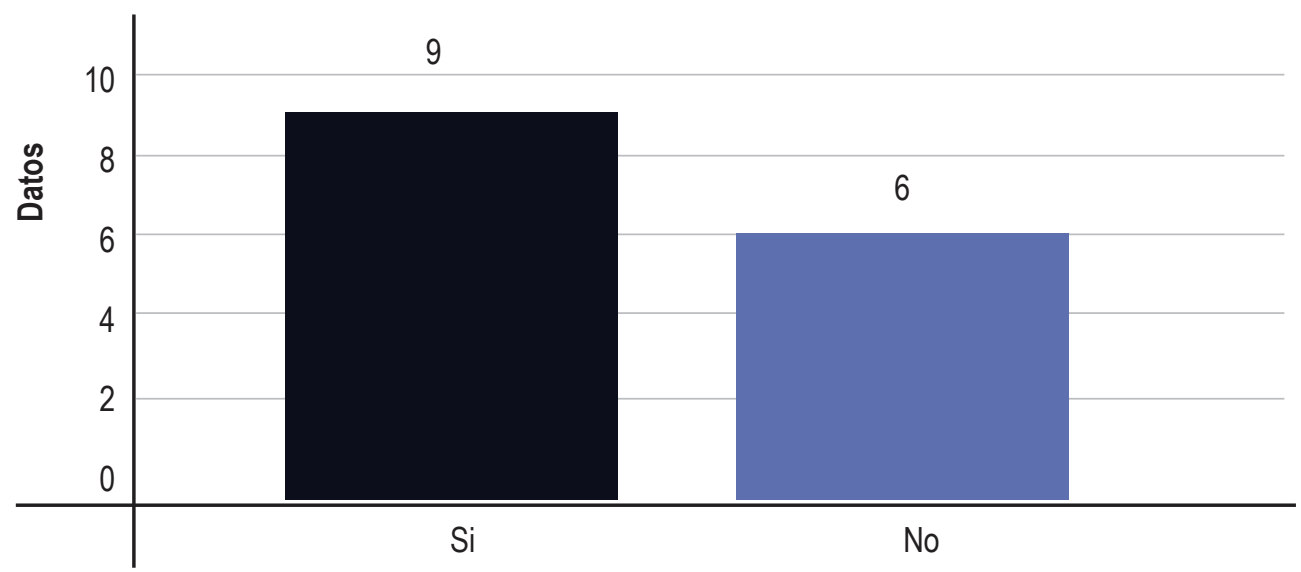

Fuente: elaboración propia.

El $56 \%$ de las empresas metalmecánicas, entrevistadas por medio de sus representantes de recursos humanos, logran cumplir sus objetivos estratégicos, pero el $44 \%$ restante no llega a cumplirlos, debido a muchos factores llegan que afectan la organización de tal manera que en ciertos casos tienen pérdidas monetarias importantes en su planificación anual.

Trece de los gerentes entrevistados sí logran encontrar un enlace entre las variables cultura organizacional y objetivos estratégicos, puesto que durante la entrevista decían estar conscientes de que al tener al capital humano conforme y cómodo trabajando, este a su vez reditúa más trabajo y, a su vez, mejor actitud para lograr las metas establecidas por las empresas. Sin embargo, esto no es garantía total de que se cumplan todos los objetivos, pues existen otros aspectos a tomar en cuenta que pueden afectar, por ejemplo aspectos de legislación de un país, lo que puede contribuir al no-cumplimiento de objetivos. 
Gráfico 5. Relación entre variables cultura organizacional y objetivos estratégicos

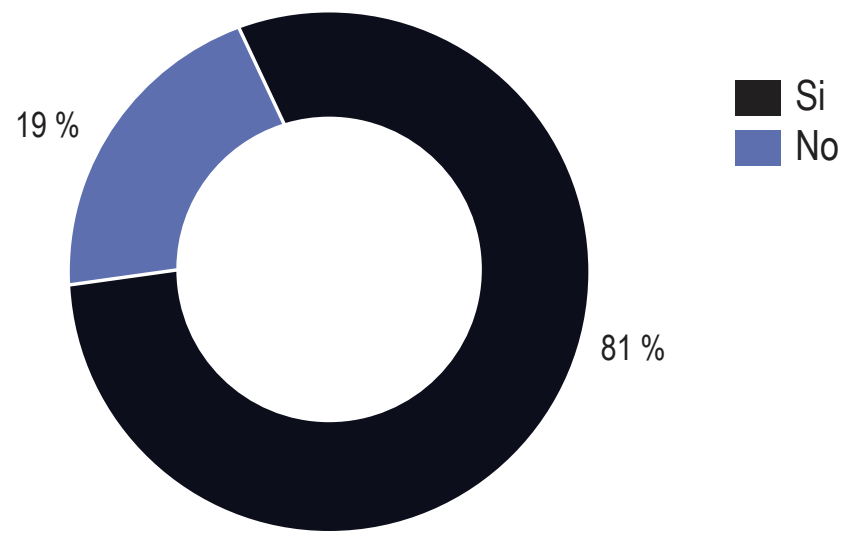

Fuente: elaboración propia.

Gráfico 6. Seguimiento entre variables: cultura organizacional y objetivos estratégicos

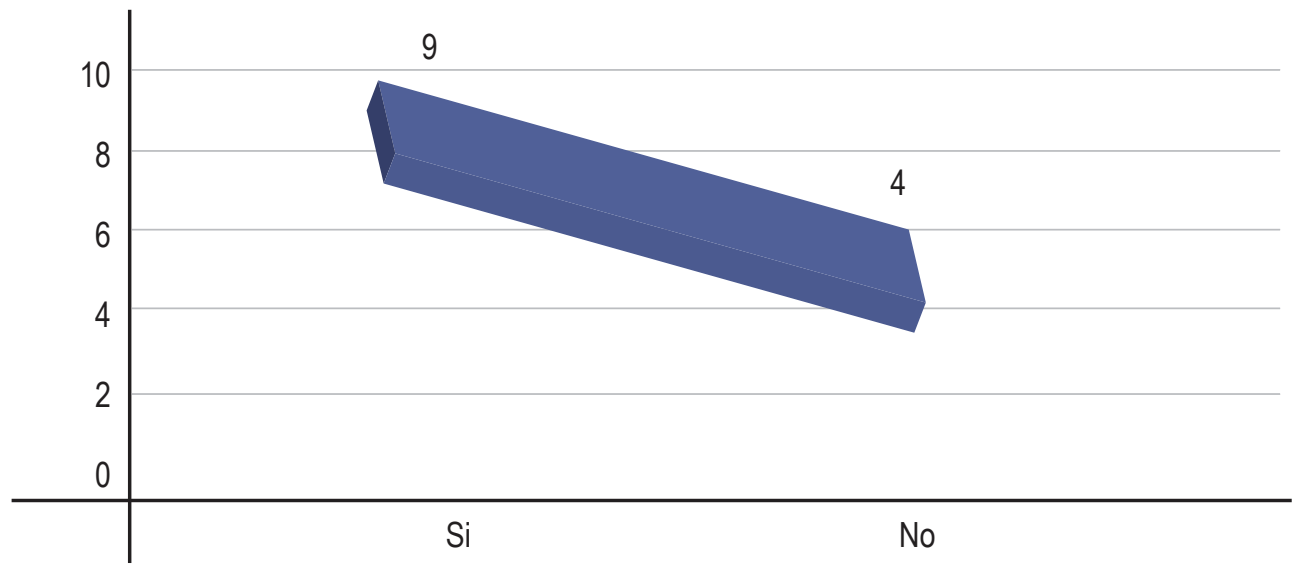

Fuente: elaboración propia.

El $56 \%$ de los encuestados indica que además de reconocer que existe un enlace entre las dos variables, se le debe de dar un seguimiento a estas, pues no se trata solamente de saber que hay una correlación, sino de conocer el grado de esa correlación; asimismo, qué tanta necesidad de atender esta correlación debe existir. Esta parte es la que se aplica a la gestión de los gerentes de recursos humanos y provee información adecuada para que se pueda dar una retroalimentación adecuada a los directivos de la organización en cuanto al seguimiento de los objetivos establecidos. 
Gráfico 7. Disposición monetaria (presupuesto) al seguimiento de las variables: cultura organizacional y objetivos estratégicos

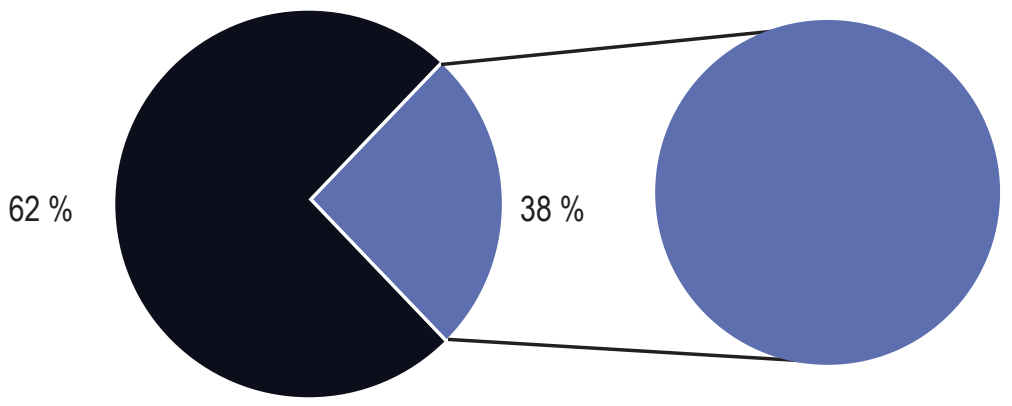

Si

Fuente: elaboración propia.

Al menos 10 de los gerentes encuestados indicaron que sí tienen un presupuesto monetario para dar seguimiento a las variables en mención, puesto que la cultura organizacional exige retroalimentación, acompañamiento y su respectiva administración, lo que representa un costo; también los objetivos organizacionales necesitan de información y de un seguimiento financiero para atender las contingencias que sucedan.

El resto de encuestados, los otros 6 gerentes, indicaron que no tienen un presupuesto como tal para atender estas demandas; en respuesta hacen algunas actividades y seguimientos que no requieren inversión, ya que sus respectivas organizaciones les dan cierta prioridad a otros rubros.

Con el análisis de los aspectos y gráficos anteriores, también se hace un llamado a la conciencia del sector laboral y personal de los trabajadores, pues no se pretende que solamente sea responsabilidad de una de las dos partes (patrono o trabajador), sino que se busca una concienciación acerca de la importancia que tiene entender la necesidad de concatenar estas dos partes y lograr lo que se plantea una empresa; pues a la larga, estos resultados son para las dos partes, están unidos estos caminos, porque al final la organización se vuelve proactiva en su espacio de desarrollo.

También se hace hincapié en que las mediciones a todo nivel en una organización son preponderantes, ya que permiten obtener información que ayuda a realinear el camino y así lograr no separarse tanto de lo que se establece en su momento. El 
capital humano es tan importante como la organización en sí misma y, si no está atendido, puede tenerse una fuga sin control con respecto al aprovechamiento de sus capacidades para la empresa.

\section{CONCLUSIONES}

La atención y el manejo de la cultura organizacional por parte de los gestores de capital humano y de la cúpula de las organizaciones, permite cumplir muchos objetivos, tales como ahorrar costos laborales y mantener una identificación del personal hacia su organización; esto facilita la sanidad laboral y que la empresa tenga una imagen sana desde su interior, la cual se podrá percibir al momento de hablar con cualquier colaborador o simplemente al momento de ver la respuesta de una empresa con respecto a sus competidores, pues serán respuestas inmediatas y bien planeadas para lograr metas importantes.

Se necesita que la gestión de los administradores del capital humano sea mucho más enfocada a la parte estratégica, pues esto permite que su gestión tenga mucha más atención por parte de la cúpula organizacional, que solamente tener una gestión operativa sin relevancia.

Al momento de enlazar estas dos variables se requiere compromiso por parte no solo del área de capital humano, sino todo un compromiso a nivel general, para que pueda ser una organización proactiva y no reactiva.

\section{AGRADECIMIENTO}

A Rafael Núñez Solórzano, Oscar Molina, Ángel Ávila y Héctor Galindo, por su colaboración en el desarrollo de la investigación. 


\section{BIBLIOGRAFÍA}

Alabart, Y. (2015). Modelo, metodología y herramientas para el diagnóstico de la cultura organizacional, resultados de investigación. III Congreso Internacional de Cultura Organizacioinal, Ecuador: Universidad Tecnológica Empresarial de Guayaquil.

Figueroa, I. (2016). Gran negocio. ¿Y dónde está el dinero? Guatemala: PF Publishing.

Gómez, I. (2013). Innovación y cultura organizacional. Bogotá: UNED.

Kovacevik, A. y Reynoso, A. (2010). El diamante de la excelencia organizacional. Chile-Guatemala: Aguilar Chilena de Ediciones.

Luissier, R. y Achúa, C. (2016). Liderazgo, teoría, aplicación y desarrollo de habilidades. México: Cengage Learning.

Paz Subillaga, A. (2015). El desempeño laboral como determinante de la productividad en la industria metalmecánica guatemalteca. Guatemala: Universidad Mariano Gálvez de Guatemala.

Sánchez, I. (2010). Desarrollo de un instrumento de medida de la cultura organizativa: un modelo confirmatorio en los hoteles españoles. Revista Europea de Dirección y Economía de Empresa, (19)1, 107-126. España.

Soares, M. (2015). La comunicación no verbal puede ser un activo para quien necesite ejercer un liderazgo. Revista A Linguagem corporal dos líderes. España. Recuperado de: file:///C:/Documents\%20and\%20Settings/TEMP/Mis\%20documentosDownloads/Dialnet-LaComunicacionNoVerbalPuedeSerUnActivoParaQuien Nec-4997124.pdf

Vilariño, Carlos. (2013). Contribución a la gestión estratégica organizacional con enfoque organizacional. Revista Venezolana de Información Tecnología y Conocimiento. Venezuela. Recuperado de: http://www.redalyc.org/articulo.oa?i$d=82326270004$

Werther, W.; Davis, K. y Guzmán, M. (2014). Administración de recursos humanos. Gestión del capital humano. México: Mc Graw Hill. 